IOS Press

\title{
Treatment of back pain in active axial spondyloarthritis with serial locoregional water-filtered infrared A radiation: A randomized controlled trial
}

\author{
Philipp Klemm ${ }^{1, *}$, Iris Aykara ${ }^{1}$, Markus Eichelmann, Elena Neumann, Klaus Frommer and Uwe Lange \\ Department of Rheumatology, Immunology, Osteology and Physical Medicine, Campus Kerckhoff, \\ Justus-Liebig-University Gießen, Bad Nauheim, Germany
}

\section{Received 11 March 2021}

Accepted 4 August 2021

\begin{abstract}
.
BACKGROUND: Axial spondyloarthritis (axSpA) is an inflammatory rheumatic disease primarily affecting the axial skeleton. OBJECTIVE: To evaluate the short-term effects of locoregional water-filtered infrared A radiation (sl-wIRAR) in the treatment of lower back pain in patients with axSpA.

METHODS: Patients with active axSpA with non-steroidal anti-inflammatory drug (NSAID) therapy undergoing a 7-day multimodal rheumatologic complex treatment in an in-patient setting were eligible. Patients were randomly assigned to the intervention group (IG) receiving sl-wIRAR treatment of the back ( 2 treatments/day for 30 min each for 6 days) or to the control group (CG) receiving no treatment. Primary outcome was a between-group difference in pain after sl-wIRAR therapy measured on a numeric rating scale (NRS) $(0=$ no pain, $10=$ worst pain). Secondary outcomes included an assessment of $\mathrm{i})$ the onset and development of analgesic effects and an evaluation of whether sl-wIRAR ii) improved axSpA-specific well-being and iii) influenced serum cytokine levels.

RESULTS: Seventy-one patients were enrolled, completed the trial and were analyzed (IG: 36 patients, CG: 35 patients). In the IG, there was a statistically significant change $(p<0.0005)$ in pain level [NRS] $(1.6 \pm 1.9$ [5; 2$])$ from baseline $(4.1 \pm 2.4[0$; $8])$ to trial completion $(2.6 \pm 2.0[0 ; 7])$ and a significant difference to the CG $(p=0.006)$. In the IG there was a significant improvement in axSpA-specific well-being (BAS-G) $(p=0.006)$. A physiologically relevant change in serum cytokine levels could not be observed.

CONCLUSION: sl-wIRAR treatment can be useful in the treatment of patients with active axSpA as it leads to a rapid reduction of pain.
\end{abstract}

Keywords: Water-filtered infrared A radiation, axial spondylarthritis, heat therapy, cytokines, physical therapy

\footnotetext{
Contributed equally.

${ }^{*}$ Corresponding author: Philipp Klemm, Department of Rheumatology, Immunology, Osteology and Physical Medicine, Campus Kerckhoff, Justus-Liebig-University Gießen, Benekestr. 2-8, $61231 \mathrm{Bad}$ Nauheim, Germany. Tel.: +496032 996 2101; Fax: +496032996 2104; E-mail: p.klemm@kerckhoff-klinik.de.
}

\section{Introduction}

Axial spondyloarthritis (axSpA) is an inflammatory rheumatic disease primarily affecting the axial skeleton with a prevalence between 0.3 and $1.4 \%$ in the general population [1]. AxSpA includes patients who have already developed structural and radiologically assessable damage (radiographic axSpA, r-axSpA, formerly called 
ankylosing spondylitis) and patients without such damage (non-radiographic axSpA, nr-axSpA) [1]. As axSpA is a chronic, non-curable disease with potential for a severe disease progression, lifelong pharmacological therapy is common $[1,2]$. Treatment goals aim to maximize health-related quality of life through control of symptoms and inflammation, prevention of progressive structural damage, preservation/normalization of function and social participation [2]. Therefore, multidisciplinary and multimodal treatment with both pharmacological and non-pharmacological treatment modalities are required [2]. Regarding pharmacological treatment, patients with active axSpA are initially treated with non-steroidal anti-inflammatory drugs (NSAIDs). For patients with inadequate response to NSAIDs, biological disease modifying anti-rheumatic drug (bDMARD) therapy can be started with tumor necrosis factor (TNF)-inhibitors and interleukin (IL)-17-inhibitors being the only two approved options $[1,2]$.

Periods of flares (clinical worsening) and remission are common for inflammatory rheumatic diseases in general and frequent in axSpA [3]. The term "flare" is poorly defined, often interpreted differently by both rheumatologists and patients and not well investigated [4]. However, a definition for a clinically relevant exacerbation in axSpA has recently been established for use in clinical trials based on the Ankylosing Spondylitis Disease Activity Score (ASDAS) [3], which may encourage further research and promote reproducibility. Nevertheless, flares seem to occur quite frequently. About $74 \%$ of patients primarily treated with NSAIDs [5] and $25 \%$ of patients primarily treated with bDMARDs reported at least one flare [6], both within a 3-month period. While not every flare is long-lasting ( $>3$ days), flares are related to a decrease in physical activity and well-being [6]. In addition, as flares are still quite common even under (stable) bDMARDtherapy [6], not every flare leads to a change in pharmacological treatment. Physical therapy (PT) could therefore offer an alternative to treat flares. Different forms and modalities of whole-body hyperthermia have shown to reduce pain and disease activity and even (pro-inflammatory) cytokine levels in axSpA [7-13]. Out of all these hyperthermia modalities, water-filtered infrared A radiation (wIRAR) showed particular potential. wIRAR is a form of infrared heat radiation in the range of 780-1400 nm with high tissue penetration and low thermal load on the skin surface, which is easy to apply in a contact-free manner [14]. The water filtering reduces the radiation components in the undesired infrared $\mathrm{B}$ and $\mathrm{C}$ range $(<5 \%)$. wIRAR showed temperature-dependent and -independent effects without relevant thermal energy transfer and/or relevant temperature changes [14]. It is therefore not only used dermatologically in acute and chronic wound healing as it promotes perfusion, alleviates pain and has anti-infectious effects [15], but is also used in oncology [16] and rheumatology [10]. Until now, wIRAR has only been used in rheumatology, specifically in the treatment of axSpA, as a whole-body treatment but not locally [10]. Whole-body wIRAR resulted in less pain and disease activity and allowed reduced analgesic usage [10]. However, whole-body wIRAR needs special equipment, that is expensive and requires a lot of space. Thus, home application is almost impossible and only a subset of hospitals and practices offer whole-body wIRAR. On the other hand, locally applied wIRAR needs only a single probe, that does not require much room, is relatively inexpensive and therefore treatment can even be used at home.

As flares are common in axSpA patients and pharmacological therapy options are limited, we investigated the effect of serial locally applied wIRAR targeted at the lower back in patients with active axSpA on pain and its development and hypothesized an analgesic treatment effect.

\section{Methods and study design}

\subsection{Trial design}

To assess short-term effects on pain of locoregional water-filtered infrared A radiation (sl-wIRAR) of the lower back in patients with active axSpA, we conducted a prospective monocentric randomized controlled trial with an assessor-blinded parallel group design. Patients were randomized using simple randomization procedures (computerized random numbers) and equally allocated to either the intervention group (IG) receiving sl-wIRAR of the lower back or the control group (CG) receiving no treatment.

\subsection{Participants}

Eligible patients were older than 18 years, had axSpA fulfilling the Assessment of Spondyloarthritis international Society (ASAS) classification criteria [17], had active disease as defined by a Bath Ankylosing Spondylitis Disease Activity Index (BASDAI) of 4 to 7 (moderate disease activity), received only NSAID therapy and/or non-pharmacological therapy that had been 
stable for more than 4 weeks prior to study start and were about to begin a 7-day multimodal rheumatologic complex treatment (MRCT) due to pain exacerbation or loss of functionality. Patients were excluded if they had a contraindication to hyperthermia (e.g. heat intolerance or intake of photosensitizing drugs), could not begin a 7-day MRCT (e.g. due to acute infections), received or previously received either systemic or local glucocorticoids or any disease-modifying anti-rheumatic drugs (DMARDs) in the previous 4 weeks.

\subsection{Setting}

The study was conducted at Campus Kerckhoff, Justus-Liebig-University Gießen, Dept. of Rheumatology, Clinical Immunology, Osteology and Physical Medicine. Approval of the local ethics committee of the Faculty of Medicine of the Justus-Liebig-University Gießen (vote no. 17/16) was obtained and all study procedures were conducted according to the Declaration of Helsinki. The study was registered on the German Clinical Trial Register (www.drks.de) under no. DRKS00021257.

\subsection{Interventions}

The study was performed during a 7-day MRCT which both the IG and CG received. MRCT is a special multimodal treatment concept in Germany with a strong emphasis on physical therapy (PT). As part of this MRCT, each participant received $11 \mathrm{~h}$ of PT with a duration of $30 \mathrm{~min} / \mathrm{PT}$ modality. To allow comparability between IG and CG, each patient received 22 PT sessions over 7 days $(7 \times$ physiotherapy, $3 \times$ pain processing strategies, $7 \times$ classic massage, $3 \times$ electrotherapy, $2 \times$ patient disease training program).

The IG received two daily applications (morning/afternoon) of sl-wIRAR of the lower back for 30 minutes, each for 6 consecutive days cumulating in a total of 12 sl-wIRAR applications. The intervention of sl-wIRAR was performed using a HydrosunVR 750 device (Hydrosun Medizintechnik GmbH, Muellheim, Germany). The radiation was strictly applied vertically in a radiation field of $25 \mathrm{~cm}$ encompassing the lower thoracic and lumbar area (see Supplementary Fig. 1). The radiation intensity was $160 \mathrm{~mW} / \mathrm{cm}^{2}$ with a radiation distance of $35 \mathrm{~cm}$. The $\mathrm{CG}$ did not receive any treatment besides MRCT.

\subsection{Outcomes and assessment}

Primary outcome was a between-group difference in pain on day 6 (in the evening) after 12 applications of sl-wIRAR therapy (2 applications/day). Pain was assessed on a numeric rating scale (NRS) $(0=$ no pain, $10=$ worst possible pain). A change greater than 2 is considered to be a clinically important improvement (MCII) [18].

Secondary outcomes included an assessment of i) the onset and development of analgesic effects of sl-wIRAR and an evaluation of whether sl-wIRAR ii) improves axSpA-specific well-being and iii) influences serum cytokine levels.

In order to assess the onset and development of analgesic effects, pain levels were measured at baseline using a NRS in the evening of day 1 after 2 sl-wIRAR applications (2 sl-wIRAR applications in total), in the morning of day 2 before sl-wIRAR treatment ( 2 slwIRAR applications in total), in the evening of day 2 after treatment (4 sl-wIRAR applications in total), in the morning of day 6 before treatment (10 sl-wIRAR applications in total) and in the evening of day 6 after treatment (12 sl-wIRAR applications in total).

Disease-specific well-being was evaluated using the Bath Ankylosing Spondylitis Patient Global Score (BAS-G) [19], which reflects the effect of axSpA on the patient's well-being by asking two questions assessing the impact of axSpA on a patient's well-being over (i) the last week and (ii) over the last six months on a $10 \mathrm{~cm}$ visual analogue scale $(0 \mathrm{~cm}=$ no impact, $10 \mathrm{~cm}=$ max. impact). The score is determined by calculating the arithmetic mean of the two analogue scale assessments. A score of 0 equals no impact of axSpA on well-being and 10 a maximum impact. An absolute difference of 1.5 or a relative difference of $20 \%$ are considered MCII for patient global assessments [20].

To investigate underlying mechanisms of the hypothesized (rapid) changes in pain, we evaluated serum cytokine levels of pro-inflammatory (IL- $1 \beta$ and IL-6) and anti-inflammatory cytokines (IL-10) using ELISA at baseline and post-intervention. Serum was centrifuged at $3.500 \mathrm{rpm}$ at $15^{\circ} \mathrm{C}$ for $10 \mathrm{~min}$. The samples were stored at $-80^{\circ} \mathrm{C}$ until further use. Cytokine levels (IL1, IL-6 and IL-10) were measured in the sera using Quantikine ${ }^{\circledR}$ ELISAs (R\&D Systems) according to the manufacturer's instructions. Optical readings were taken with a SUNRISE (TECAN) system at $450 \mathrm{~nm}$ and $570 \mathrm{~nm}$ for wavelength correction. Additionally, this study recorded sl-wIRAR-related adverse and severe adverse events.

\subsection{Sample size calculation}

Based on the reported effects of whole-body hyperthermia using whole-body wIRAR in axSpA [12] we 


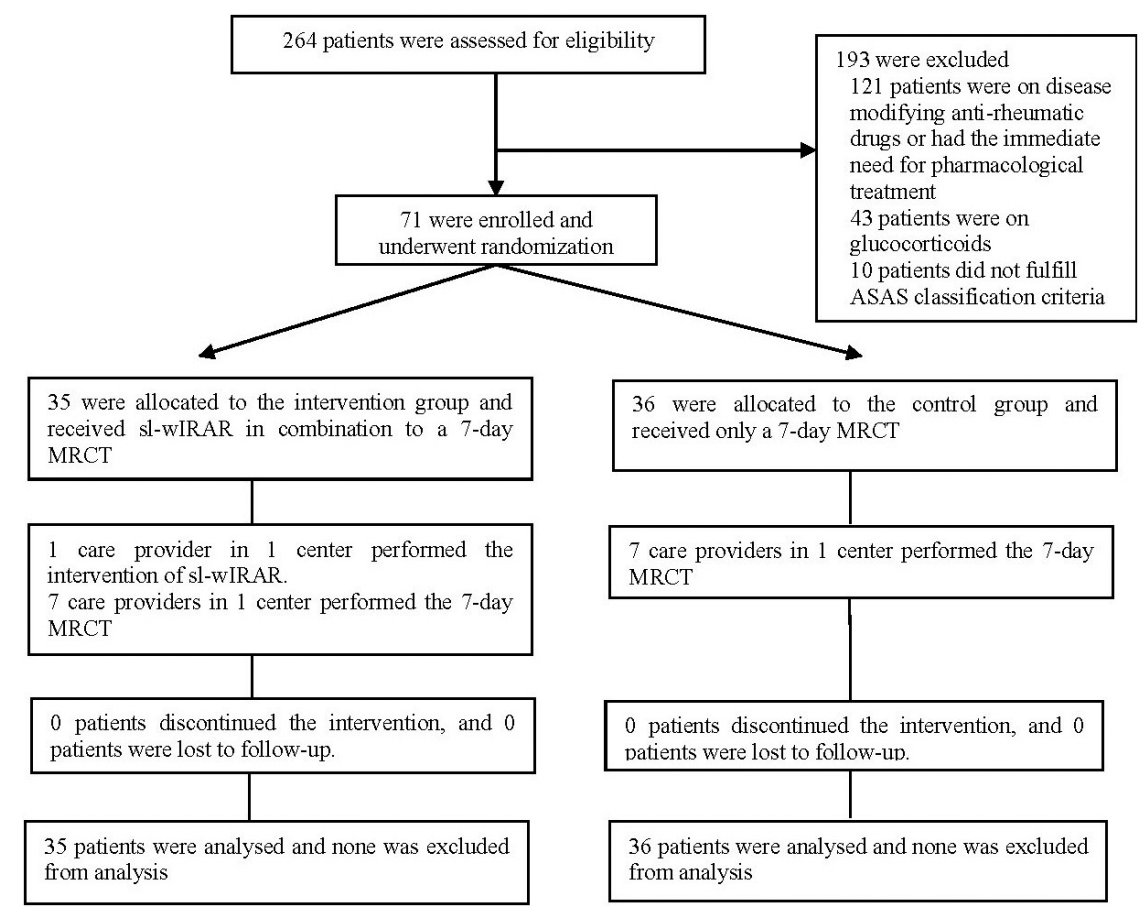

Fig. 1. Trial enrollment and follow-up.

aimed to recruit 35 patients per sequence group with a dropout rate of approximately $20 \%$. Calculations were performed in nQuery 8.

\subsection{Statistics}

Data are listed descriptively using mean and standard deviation (SD). Differences are displayed using mean and standard error (SE). The hypothesis of normality was tested with Q-Q plots and the Shapiro-Wilk test and subsequently refuted. Intra-group differences were assessed by the non-parametric Wilcoxon test, betweengroup differences by the non-parametric Mann-Whitney U test. Bonferroni's method was used to correct for multiple comparisons. The multiple alpha level of the study was set to 0.05 . Calculations were performed using IBM SPSS Statistics V.20 Windows (SPSS Inc, Chicago, Illinois, USA).

\section{Results}

Between June 01, 2017 and June 01, 2018, 264 SpA patients were assessed for eligibility, of which 193 were excluded. $71 \mathrm{SpA}$ patients with active disease and an exacerbation of pain, in parallel receiving a 7-day MRCT, were enrolled and randomized equally to either the in-
Table 1

Patient and disease characteristics at baseline

\begin{tabular}{lll}
\hline & \multicolumn{1}{c}{$\begin{array}{c}\text { Intervention group } \\
(n=36)\end{array}$} & \multicolumn{1}{c}{$\begin{array}{c}\text { Control group } \\
(n=35)\end{array}$} \\
\hline Age (years) & $51.7 \pm 10.8$ & $51.1 \pm 10.2$ \\
Disease duration & $5.8 \pm 4.2$ & $6.1 \pm 4.5$ \\
Sex (female/male) & $24(66 \%) / 12(34 \%)$ & $24(68 \%) / 11(32 \%)$ \\
HLA-B27 positivity & $30(83 \%)$ & $32(91 \%)$ \\
BASDAI & $4.8 \pm 1.8$ & $4.6 \pm 2.1$ \\
BASFI & $4.1 \pm 2.2$ & $4.5 \pm 2.6$ \\
NSAIDs & $34(94 \%)$ & $32(91 \%)$ \\
\hline
\end{tabular}

tervention group (IG) receiving sl-wIRAR of the lower back or the control group (CG) receiving no treatment. Seventy-one patients completed the trial. The trial design is shown in Fig. 1.

Mean age in both groups was 51 years with a disease duration of 5.8 in the IG and 6.1 years in the CG. Disease activity and disease-related functional capacity and disability were comparable between groups. Further patient and disease characteristics are listed in Table 1.

The primary outcome of this study was met with a statistically significant between-group difference in pain after intervention (95\% confidence interval (CI), -2.8 to $-0.8, p=0.006)$. The IG experienced a statistically significant improvement of $-1.6 \pm 0.3$ (mean \pm standard error (SE)) from baseline to after intervention (95\% CI, -2.2 to $-0.9, p<0.001)$, while the control 
Table 2

Change in pain levels (NRS) between baseline and after trial completion

\begin{tabular}{lclll}
\hline & Baseline & After intervention & Difference & \multicolumn{1}{c}{$p$-value $(95 \% \mathrm{CI})$} \\
\hline IG $(n=36)$ & $4.1 \pm 2.4$ & $2.6 \pm 2.0$ & $-1.6 \pm 0.3$ & $<0.001(-2.2$ to -0.9$)$ \\
CG $(n=35)$ & $4.8 \pm 2.5$ & $4.4 \pm 2.2$ & $-0.3 \pm 0.2$ & $0.088(-0.8$ to 0.1$)$ \\
$p$-value $(95 \% \mathrm{CI})$ & & $0.006(-2.8$ to -0.8$)$ & & \\
\hline
\end{tabular}

Mean \pm standard deviation are shown for values at specific time points. Differences are displayed using mean \pm standard error. IG: intervention group, CG: control group, CI: confidence interval.

Table 3

Onset and development of sl-wIRAR effects on pain levels

\begin{tabular}{|c|c|c|c|}
\hline & $\begin{array}{c}\text { Intervention group } \\
(n=36)\end{array}$ & $\begin{array}{c}\text { Control group } \\
(n=35)\end{array}$ & $p$-value** \\
\hline Baseline (day 1 before treatment) & $4.1 \pm 2.4$ & $4.8 \pm 2.5$ & \\
\hline Day 1 after treatment & $3.5 \pm 2.2$ & $4.7 \pm 2.3$ & \\
\hline Difference ( $p$-value ${ }^{*}$ ) & $-0.7 \pm 1.2(p<0.001)$ & $-0.1 \pm 0.6(p<0.405)$ & $p<0.001$ \\
\hline Day 2 before treatment & $3.9 \pm 2.2$ & $4.8 \pm 2.2$ & \\
\hline Day 2 after treatment & $3.3 \pm 2.2$ & $4.8 \pm 2.2$ & \\
\hline Difference ( $p$-value*) & $-0.6 \pm 1.1(p<0.005)$ & $-0.0 \pm 0.7(p<0.796)$ & $p<0.007$ \\
\hline Day 5 before treatment & $3.6 \pm 2.3$ & $4.8 \pm 2.1$ & \\
\hline Day after treatment & $3.1 \pm 2.2$ & $4.5 \pm 2.1[1 ; 8]$ & \\
\hline Difference ( $p$-value ${ }^{*}$ ) & $-0.5 \pm 0.9(p=0.003)$ & $-0.3 \pm 0.9(p=0.032)$ & $p=0.747$ \\
\hline Day 6 before treatment & $3.3 \pm 2.4$ & $4.6 \pm 2.3$ & \\
\hline Day 6 after treatment (trial completion) & $2.6 \pm 2.0$ & $4.4 \pm 2.2$ & \\
\hline Difference $\left(p\right.$-value $\left.{ }^{*}\right)$ & $-0.7 \pm 1.0(p<0.0005)$ & $-0.2 \pm 0.6(p<0.109)$ & $p<0.023$ \\
\hline
\end{tabular}

Pain levels (NRS) were assessed on days 1,2,5 and 6 before and after treatment ( 2 applications of wIRAR of the lower back per day, 12 applications in total). Mean \pm standard deviation are shown. ${ }^{*} p$-values of the Wilcoxon test for intra-group differences to compare two related samples. ${ }^{* *} p$-values of the Mann-Whitney test for between-group differences in differences between both treatment arms.

Table 4

Assessment of cytokine levels of IL-1ß, -6 , and -10 over time

\begin{tabular}{llcclc}
\hline & Treatment arm & Day 1 & Day 6 & Day 6-Day 1 & $p$-value* \\
\hline IL-1ß & IG $(n=36)$ & $0.3 \pm 1.1$ & $0.3 \pm 1.0$ & $-0.0 \pm 0.1$ & $p<0.317$ \\
& CG $(n=35)$ & $1.1 \pm 0.6$ & $1.3 \pm 0.7$ & $0.1 \pm 0.8$ & $p<0.694$ \\
& $p$-value** & & & $p<0.695$ & \\
IL-6 & IG $(n=36)$ & $3.4 \pm 5.7$ & $2.5 \pm 3.2$ & $-0.9 \pm 5.8$ & $p<0.904$ \\
& CG $(n=35)$ & $3.9 \pm 3.6$ & $3.5 \pm 4.4$ & $-0.4 \pm 2.4$ & $p<0.133$ \\
& $p$-value** & & & $p<0.137$ & \\
IL-10 & IG $(n=36)$ & $7.3 \pm 17.6$ & $4.3 \pm 5.2$ & $-3.0 \pm 18.0$ & $p<0.401$ \\
& CG $(n=35)$ & $6.4 \pm 4.8$ & $4.5 \pm 6.9$ & $-1.9 \pm 8.2$ & $p<0.054$ \\
& $p$-value** & & & $p<0.117$ & \\
\hline
\end{tabular}

Values are presented as mean \pm standard deviation. IG: intervention group, CG: control group, IL: interleukin. ${ }^{*} p$-values of the Wilcoxon test for intra-group differences to compare two related samples. ${ }^{* *} p$-values of the Mann-Whitney test for between-group differences in differences between both treatment arms.

group experienced a nominal improvement of $-0.3 \pm$ 0.2 (95\% CI, -0.8 to $0.1, p=0.088$ ) (Table 2 ).

As secondary outcomes, we further investigated the effects of sl-wIRAR (2 applications/day over 6 days with 12 applications in total) on pain.

Onset of a statistically significant analgesic effect of sl-wIRAR treatment could be seen immediately after 2 applications on the evening of day 1 in the IG with a statistically significant between-group difference. From there on, statistically significant differences in the IG (before and after treatment) and between groups were found in each comparison. Analgesic effects did not vanish after night rest at day 2 and increased in a cumulating manner as pain values steadily decreased at each reading (Table 3 ).

BAS-G, which reflects the effect of axSpA on the patient's well-being, was statistically changed in the IG with a mean difference $( \pm$ SD) of $-0.5 \pm 1.1$ ( $p=0.006$ ), while the CG missed statistically significance $(p=0.051)$. There was no statistically significant between-group difference (Supplementary Table 1).

Assessed serum cytokine levels of pro-inflammatory 
(IL-1 and IL-6) and anti-inflammatory cytokines (IL10) did not show any physiologically relevant or statistically significant changes (Table 4).

No adverse events related to WIRAR were recorded in this study.

\section{Discussion}

To the best of our knowledge, this is the first randomized controlled trial to investigate the effects of slwIRAR on pain, its onset and development over time in axSpA patients. This study was able to demonstrate that sl-wIRAR treatment is effective and has a significant analgesic effect that is measurable after only 2 applications and increases with further treatment. With only 6 treatment days with 2 applications/day, the treatment group showed a statistically significant improvement in pain and BAS-G in comparison to the non-treatment group. However, with a mean change of -1.5 in pain levels, a clinically significant improvement could not be measured. Only patients with active axSpA were eligible for this trial. Thus, baseline BASDAI (mean: 4.8 IG and 4.6 CG), BASFI (mean: 4.1 IG and 4.5 CG) and pain levels (mean: 4.1 IG and 4.8 CG) were elevated. As flares, although poorly-defined outside of clinical trials [4], are common in axSpA patients treated primarily with bDMARDs [6] and in patients treated with NSAIDs [5], sl-wIRAR treatment seems to be a good alternative to a change in pharmacological therapy. Especially, when flare duration is variable, a complementary and easy to apply sl-wIRAR treatment that can be performed in an outpatient setting seems to be suitable for initial treatment with a focus on a quick pain reduction.

The rapid onset of pain relief lasting for up to 6 days is based on thermal and non-thermal effects. The thermal effect results from increased blood flow and improved elimination of accumulated metabolites including pain mediators as well as increased metabolism due to the increased tissue temperature. The non-thermal effect results from a direct effect on cellular structures and cells as well as altered muscle toning with consecutive pain reduction [14]. The pro-inflammatory cytokines IL-1 $\beta$, IL- 6 and TNF- $\alpha$ play a central role in both, the inflammatory process and the inflammation induced pain [21]. Local nociceptive reactions involve peripheral polymodal nociceptors expressing glycoprotein 130 (gp 130), which plays a role in cytokine signaling [22,23]. In addition, proinflammatory cytokines induce systemic effects. For example, IL-6 and PGE 2 are regulators of the hepatic synthesis of $\mathrm{C}$-reactive protein [21]. A distinction is made between hyperalgesic mediators, e.g. prostaglandins, IL-1, -6, -8, TNF$\alpha$, and analgesic mediators such as IL-1, -4, -10, -13. During inflammatory pain, the cytokine interplay is prominent: In the early stage, hyperalgesic mediators dominate while at the same time analgetically active cytokines are induced by the immune system [22,23]. A decrease of these mediators may lead to reduced depolarization of the peripheral nociceptors due to reduced input from ascending neurons in the cortical pain matrix and therefore enhance a consecutive decrease in pain sensation.

During inflammation, the nociceptors of the joints are sensitized to mechanical stimuli and usually mute sensory C-fibers become mechanosensitive [24]. A decrease in inflammatory mediators could thus influence this process.

Proinflammatory cytokines induce the production of nerve growth factor (NGF) [24] which activates and sensitizes tropomysin receptor kinase (TrkA)-positive sensitive neurons to mechanical, chemical and thermal stimuli and changes the properties of $\mathrm{A} \delta$ fibers (sensitation). A blockade of NGF-TrkA causes a reduction of skeletal pain [24]. It is possible that a decrease of the proinflammatory cytokines reduces the NGF production with consecutive desensitization of TrkA-positive sensitive neurons. However, no significant changes in the cytokines IL-1, -6 and -10 were detected in the present study after sl-wIRAR's for 6 days. Thus, we assume that recorded effects of sl-wIRAR are mainly due to thermal-effects.

Similar clinical results regarding pain were obtained by whole-body hyperthermia with water-filtered infrared A [10,25-28]. However, in comparison to wholebody wIRAR application, locoregional application is a contact-free, consumable-free, easy-to-use procedure with a good depth effect ("diathermy"). It has a mild effect on the circulation and can be used for treatment in different positions. In addition, it can be easily dosed individually by varying the irradiation distance and thus the irradiation intensity and the duration of the application. Sl-wIRAR can thus be regarded as a sensible and effective addition to any multimodal treatment concept in $\operatorname{axSpA}$.

\subsection{Limitation}

A limitation of this trial is the lack of a follow-up period. We cannot say how long the analgesic effects observed after 6 days of treatment last. Since this trial fo- 
cused on acute pain exacerbations and flares, the acute therapy and its effect as well as the onset and evolution of analgesic effects were of interest. Common sense dictates that a one-time intervention for a chronic disease such as axSpA is unlikely to result in long-time positive outcomes. However, the exact duration of effects after discontinued therapy remains unclear. In addition, a future trial should try to blind patients as well as assessor rather than only the assessor although this might be difficult to achieve for hyperthermic interventions.

\subsection{Generalizability}

This study is consistent with effects of hyperthermia in axSpA, particularly using whole-body hyperthermia infrared A [7,8,10,14,15]. An effect of locoregional slwIRAR on pain in axSpA patients can be derived from the results of this study.

\section{Conclusion}

Sl-wIRAR is an effective treatment option to reduce pain in axSpA with rapid onset and a cumulative beneficial effect with each use as shown over six days. Therefore, it could be a valid option to treat acute flares in axSpA patients in addition to pharmacological therapy.

\section{Acknowledgments}

The authors are grateful to patients and hospital staff who contributed to this study. They acknowledge the statistical support of MoReData GmbH, Kerkrader Strasse 11, 35394 Gießen, Germany. They also thank Ms. Carina Schreiyäck for excellent technical assistance in performing the ELISA measurements.

\section{Author contributions}

All authors contributed to the study conception and design. Material preparation, data collection and analysis were performed by Philipp Klemm, Iris Aykara, Markus Eichelmann, Elena Neumann, Klaus Frommer and Uwe Lange. The first draft of the manuscript was written by Philipp Klemm and Iris Aykara and all authors commented on previous versions of the manuscript. All authors read and approved the final manuscript.

\section{Conflict of interest}

Philipp Klemm declares no conflicts of interest. Markus Eichelmann declares no conflicts of interest. Iris Aykara declares no conflicts of interest. Elena Neumann declares no conflicts of interest. Klaus Frommer declares no conflicts of interest. Uwe Lange declares no conflicts of interest.

\section{Funding}

This study was supported by the Dr. med. h.c. Erwin Braun Foundation, Basel, a charitable, non-profit Swiss scientific foundation approved by the Swiss Federal Administration. The foundation supports clinical investigation of water-filtered infrared-A. The foundation was not involved in any content- or decision-related aspect of the study.

\section{Supplementary data}

The supplementary files are available to download from http://dx.doi.org/10.3233/BMR-210068.

\section{References}

[1] Sieper J, Poddubnyy D. Axial spondyloarthriti. Vol. 390, The Lancet. Lancet Publishing Group; 2017. 73-84.

[2] Van Der Heijde D, Ramiro S, Landewé R, Baraliakos X, Van DenBosch F, Sepriano A, et al. 2016 update of the ASASEULAR management recommendations for axial spondyloarthritis. Ann Rheum Dis. 2017 Jun 1; 76(6): 978-91.

[3] Molto A, Gossec L, Meghnathi B, Landewé RBM, van der Heijde D, Atagunduz P, et al. An Assessment in SpondyloArthritis International Society (ASAS)-endorsed definition of clinically important worsening in axial spondyloarthritis based on ASDAS. Ann Rheum Dis. 2018 Jan 1; 77(1): 124-7.

[4] Keat ACS. Axial spondyloarthritis flares - Whatever they are. Vol. 44, Journal of Rheumatology. Journal of Rheumatology; 2017. 401-3.

[5] Jacquemin C, Maksymowych WP, Boonen A, Gossec L. Patient-reported flares in ankylosing spondylitis: A crosssectional analysis of 234 patients. J Rheumatol. 2017 Apr 1; 44(4): 425-30.

[6] Jacquemin C, Molto A, Servy H, Sellam J, Foltz V, Gandjbakhch F, et al. Flares assessed weekly in patients with rheumatoid arthritis or axial spondyloarthritis and relationship with physical activity measured using a connected activity tracker: A 3-month study. RMD Open. 2017 Jun 29; 3(1): e000434.

[7] Lange U, Dischereit G. Effects of different iterative wholebody hyperthermia on pain and cytokines in rheumatic diseases: A current review. Aktuelle Rheumatol. 2018 Dec 20; 57(06): 479-83. 
[8] Tarner IH, Müller-Ladner U, Uhlemann C, Lange U. The effect of mild whole-body hyperthermia on systemic levels of TNF-alpha, IL-1beta, and IL-6 in patients with ankylosing spondylitis. Clin Rheumatol. 2009 Apr 17; 28(4): 397-402.

[9] Dischereit G, Neumann N, Müller-Ladner U, Kürten B, Lange U. The impact of serial low-dose radon hyperthermia exposure on pain, disease activity and pivotal cytokines of bone metabolism in ankylosing spondylitis - a prospective study. Aktuelle Rheumatol. 2014 Aug 4; 39(05): 304-9.

[10] Lange U, Müller-Ladner U, Dischereit G. Effectiveness of whole-body hyperthermia by mild water-filtered infrared a radiation in ankylosing spondylitis - a controlled, randomised, prospective study. Aktuelle Rheumatol. 2017 Apr 1; 42(2): 122-8.

[11] Moder A, Hufnagl C, Lind-Albrecht G, Hitzl W, Hartl A, Jakab M, et al. Effect of combined Low-Dose Radon-and Hyperthermia Treatment (LDRnHT) of patients with ankylosing spondylitis on serum levels of cytokines and bone metabolism markers: A pilot study. Int J Low Radiat. 2010 Dec; 7(6): 423-35.

[12] Dischereit G, Goronzy JE, Müller-Ladner U, Fetaj S, Lange U. Effects of serial mud baths on inflammatory rheumatic and degenerative diseases. Z Rheumatol. 2019 Mar 1; 78(2): 143-54.

[13] Lange U, Neumann N, Kürten B, Müller-Ladner U, Tarner IH. Einfluss einer seriellen niedrig dosierten RadonstollenHyperthermie auf zentrale Zytokine des Knochenmetabolismus bei ankylosierender Spondylitis. Phys Medizin Rehabil Kurortmedizin. 2012 Aug 22; 22(4): 203-6.

[14] Hoffmann G. Clinical applications of Water-Filtered InfraredA (wIRA) - a review. Phys Medizin Rehabil Kurortmedizin. 2017 Oct 1; 27(5): 265-74.

[15] Hoffmann G, Harte M, Mercer JB. Heat for wounds - Waterfiltered infrared-a (wIRA) for wound healing - a review. Vol. 14, GMS German Medical Science. Association of the Scientific Medical Societies in Germany; 2016.

[16] Notter M, Thomsen AR, Nitsche M, Hermann RM, Wolff HA, Habl G, et al. Combined wIRA-hyperthermia and hypofractionated re-irradiation in the treatment of locally recurrent breast cancer: Evaluation of therapeutic outcome based on a novel size classification. Cancers (Basel). 2020 Mar 6; 12(3): 606.

[17] Rudwaleit M, van der Heijde D, Landewe R, Listing J, Akkoc $\mathrm{N}$, Brandt J, et al. The development of Assessment of SpondyloArthritis international Society classification criteria for axial spondyloarthritis (part II): Validation and final selection. Ann Rheum Dis. 2009 Jun 1; 68(6): 777-83.
[18] Salaffi F, Stancati A, Silvestri CA, Ciapetti A, Grassi W. Minimal clinically important changes in chronic musculoskeletal pain intensity measured on a numerical rating scale. Eur J Pain. 2004 Aug; 8(4): 283-91.

[19] Jones SD, Steiner A, Garrett SL, Calin A. The bath ankylosing spondylitis patient global score (BAS-G). Br J Rheumatol. 1996; 35(1): 66-71.

[20] Tubach F, Ravaud P, Martin-Mola E, Awada H, Bellamy N, Bombardier C, et al. Minimum clinically important improvement and patient acceptable symptom state in pain and function in rheumatoid arthritis, ankylosing spondylitis, chronic back pain, hand osteoarthritis, and hip and knee osteoarthritis: Results from a prospective multina. Arthritis Care Res (Hoboken). 2012 Nov 1; 64(11): 1699-707.

[21] Rittner HL, Brack A, Stein C. Pain and the immune system: friend or foe? Vol. 51, Anaesthesist. 2002. 351-8.

[22] Boettger MK, Hensellek S, Richter F, Gajda M, Stöckigt R, von Banchet GS, et al. Antinociceptive effects of tumor necrosis factor $\alpha$ neutralization in a rat model of antigen-induced arthritis: Evidence of a neuronal target. Arthritis Rheum. 2008 Aug; 58(8): 2368-78.

[23] Kulkarni B, Bentley DE, Elliott R, Julyan PJ, Boger E, Watson A, et al. Arthritic pain is processed in brain areas concerned with emotions and fear. Arthritis Rheum. 2007 Apr; 56(4): 1345-54.

[24] Peuker E. Neuroanatomische grundlagen des gelenkschmerzes. Aktuelle Rheumatol. 2016 Aug 31; 41(04): 300-5.

[25] Lange U, Dischereit G. Effects of different iterative wholebody hyperthermia on pain and cytokines in rheumatic diseases: A current review. Aktuelle Rheumatol. 2018 Dec 20; 57(06): 479-83.

[26] Lange U, Goronzy J-E, Müller-Ladner U, Frommer K, Dischereit G. Anti-inflammatory effect of serial mud baths in rheumatoid arthritis and ankylosing spondylitis. IOSR J Pharm Biol Sci. 2018; 13(2): 59-63.

[27] Lange U, Schwab F, Muller-Ladner U, Dischereit G. Effectiveness of whole-body hyperthermia by mild water-filtered infrared a radiation in psoriatic arthritis: A controlled, randomised, prospective trial. Aktuelle Rheumatol. 2014 Jul 9; 39(05): 310-6

[28] Kern PM. Entzündung und schmerz. Aktuelle Rheumatol. 2016 Aug 31; 41(4): 306-9. 